\title{
Ecology of megabenthic bivalve communities from sandy beaches on the south coast of Portugal
}

\author{
MARTA M. RUFINO ${ }^{1,2,3}$, MIGUEL B. GASPAR ${ }^{1}$, ALEXANDRE M. PEREIRA ${ }^{1}$, \\ FRANCESC MAYNOU ${ }^{2}$ and CARLOS C. MONTEIRO ${ }^{1}$ \\ ${ }^{1}$ Instituto Nacional dos Recursos Biológicos, I.P. (INRB)/ IPIMAR, Avenida 5 de Outubro s/n, P-8700-305 Olhão, Portugal. \\ 2 Institut de Ciències del Mar (CSIC), Pg. Marítim de la Barceloneta, 37-49, 08003 Barcelona, Spain. \\ ${ }^{3}$ Present address: LNEG (INETI) Departamento de Geologia Marinha, Estrada da Portela, Zambujal, 2721-866 Alfragide, \\ Portugal. E-mail: marta.m.rufino@gmail.com
}

SUMMARY: Ecological aspects of 75 megabenthic bivalve species found on the south coast of Portugal (from Vila Real to Sagres) were studied based on the information provided by seasonal bivalve surveys from 2000 to 2007 ( 8600 hauls, simultaneously using a razor shell dredge and a clam dredge). Of the 75 species identified, 36 occurred less than 30 times, and thus were considered rare in the area. The remaining 39 bivalves were analysed according to their occurrence, bathymetric distribution, geographic preferences, seasonal preferences, burying-depth, beach exposure and river mouth proximity. All species belonging to the Pharidae and Solenidae families (razor shells) and most Tellinidae were significantly more frequent in the razor shell dredge (indicating deeper burying in the sediment), whilst the families Cardiidae and Donacidae were significantly more frequent in the clam dredge (indicating that these species are probably closer to the sediment surface). As for the season, most bivalve species occurred similarly in both seasons (19 sp; 49\%), but a large proportion were more abundant during the autumn surveys (17 sp., 44\%), whereas only three species were commoner during the spring surveys. Most species belonging to the families Cardiidae and Mactridae were commoner in the autumn surveys. The spatial distribution differed between species and cluster analysis identified four communities with greater geographic affinity. Species belonging to the family Cardiidae were preferably found in the western part (WB) and the eastern part (S) of the study area, whereas the families Donacidae, Mactridae and Tellinidae occurred mainly in the central area (EB) and the eastern (S) coastal sectors. Overall, shallower species (modal depth at 3-6 m) showed greater occurrences and abundances than the deeper ones, and the depth pattern observed did not change between seasons. Donacidae and Mactridae (except Mactra glauca) were represented essentially by shallow species, whereas Cardiidae (except Acanthocardia tuberculata and Cerastoderma edule) included mostly species occurring in deeper waters. Bathymetric sequence of species distribution within some families was observed.

Keywords: bivalves, geographic distribution, bathymetric distribution, beach morphology, river proximity.

RESUMEN: ECOLOGÍA DE COMUNIDADES DE BIVALVOS MEGABENTÓNICOS EN PLAYAS DE ARENA DE LA COSTA MERIDIONAL DE PoRTugal. - Se estudiaron algunos aspectos ecológicos de 75 especies de bivalvos megabentónicos en la costa meridional de Portugal (desde Vila Real a Sagres), basados en la información obtenida a lo largo de varios muestreos estacionales realizados entre el año 2000 y 2007 (8600 muestras). Las muestras se obtuvieron utilizando simultáneamente un rastro para capturar navajas y un rastro para capturar almejas). De los 75 taxones identificados, 36 aparecieron menos de 30 veces, considerándose raros en el área. De los 39 taxones de bivalvos restantes se analizaron los patrones de presencia, distribución batimétrica, preferencias geográficas y estacionalidad y se relacionaron con la profundidad de enterramiento, la exposición de las playas y la proximidad a desembocaduras de ríos. Todas las especies de las familias Pharidae, Solenidae (navajas) y la mayor parte de Tellinidae fueron más frecuentes en el rastro para capturar navajas, indicando una mayor profundidad de enterramiento en el sedimento, mientras que las familias Cardiidae y Donacidae fueron significativamente más frecuentes en el rastro para capturar almejas, indicando que estas especies se encuentran más cercanas a la superficie del sedimento. La mayoría de especies presentaron abundancias similares a lo largo de todo el año (19 especies, 49\%), pero una gran proporción fueron más abundantes en los muestreos de otoño (17 especies, 44\%), mientras que sólo 3 especies fueron más comunes en primavera. La mayoría de especies de las familias Cardiidae y Mactridae fueron más comunes en otoño. La distribución espacial fue distinta para cada especie y el análisis de clasificación jerárquica mostró 4 comunidades con mayor afinidad geográfica. Las especies pertenecientes a la familia Cardiidae se encontraron preferentemente en la parte occidental de la zona de estudio (WB) y en la oriental, mientras que las familias Donacidae, Mactridae y Tellinidae aparecieron principalmente en 
los sectores central (EB) y oriental (S) del área. Las especies de distribución más somera (profundidad modal 3-6 m) fueron más frecuentes y abundantes en general que las especies de más profundidad y este patrón no cambió entre primavera y otoño. Donacidae y Mactridae (excepto Mactra glauca) estuvieron representadas fundamentalmente por especies someras, mientras que los Cardiidae (excepto Acanthocardia tuberculata y Cerastoderma edule) estuvieron representados principalmente por especies de profundidad. En algunas familias se observó una distribución batimétrica secuencial.

Palabras clave: bivalvos, distribución geográfica, distribución batimétrica, morfología de playas, proximidad a ríos.

\section{INTRODUCTION}

Faunistic studies provide essential tools for understanding the dynamics of exploited communities and accordingly other inter-specific relationships (Pearson and Mannvik, 1998; Teske and Wooldridge, 2001; Abelló et al., 2002; Laudien et al., 2007). Benthic communities have long been recognised as a good tool for monitoring either man-induced or natural long-term changes in marine ecosystems (Grémare et al., 1998). Coastal areas, which are particularly vulnerable due to the impact of tourism and associated activities, have a crucial importance to the communities and thus their conservation and management should be a priority.

Marine environmental impact/monitoring studies are usually done through macrofauna sampling, which involves expensive and laborious procedures (Thrush et al., 1994; Chícharo et al., 2002; Fugi, 2007; McBreen et al., 2008). Large bivalves are easier to sample and identify, they can be good biological indicators (Simboura and Zenetos, 2002) and many species are relatively short living (3-10 years), thus rapidly reflecting environmental changes (Guillou and Sauriau, 1985; Gaspar et al., 1999b; Zeichen et al., 2002). Additionally, bivalves constitute a large fraction of the littoral benthic communities and play essential roles in ecosystems as agents of sediment structuring (Reise, 2002), as a food item of many species (Ansell et al., 1999), and as contributors to biogenic sediment formation. Long term data series can provide valuable information on species ecology through the analysis of individual occurrences in relation to environmental variables.

The beach morphodynamics (breaking waves, tides and sediment) determine the morphology and slope of the beach as well as the circulation pattern (Harriague et al., 2006) and have a strong influence on the communities living there (McLachlan, 1990). Exposed sandy beaches support benthic fauna welladapted to the physical rigours of these dynamic environments (McLachlan et al., 1995). These communities may be described by the auto-ecological hypothesis (Noy-Meir, 1979; McLachlan, 1990), which states that sandy beaches are physically controlled environments, where communities are structured by the independent responses of individual species to the physical environment, biological interactions being minimal (Defeo and McLachlan, 2005). Thus, by identifying the factors controlling individual species and determining their ecological response, one obtains a picture of the community (McLachlan et al., 1995). Among the main factors affecting sandy beach benthic fauna are beach morphology and river proximity (Dexter, 1992; Defeo and McLachlan, 2005; Cravo et al., 2006), which, though they do not constitute the environmental forces per se, are relatively easy to obtain and thus can be considered in most studies. Therefore the individual response of bivalve species to these aspects can be important for fisheries management and coastal areas protection.

A locally important bivalve dredge fishery takes place along the south coast of Portugal, targeting four species: Chamelea gallina, Spisula solida, Donax trunculus and Ensis siliqua. In order to manage this fishery, the Portuguese Institute for Fisheries Research (IPIMAR) has carried out bi-annual bivalve surveys since 1986, covering all sandy bottoms along the southern Portugal from the Mediterranean areas to the Atlantic ones (Gaspar, 1996; Gaspar et al., 1999a; Rufino et al., 2008). These surveys are a unique opportunity to delimit the geographic and bathymetric distribution and interrelationships of many species with a high degree of accuracy, given the duration of the surveys and the high sampling effort. Information on beach profile is available only for a small part of the surveyed area: some sites off the eastern part of the Algarve south coast (west side of the Ria Formosa: Garrão, Faro and Culatra, eastern south coast of Portugal), all showing a reflective profile with a steep upper foreshore and a more gentle low-tide terrace (Ciavola et al., 1997; Reyes et al., 1999). The predominant wave direction in the area is W/SW (90\% of the year) (Costa, 1994). Wave height ranges from $0.30 \mathrm{~m}$ to $1.8 \mathrm{~m}$, with rare exceptional heights of more than $3.7 \mathrm{~m}$. Such high waves are associated with storms from the $\mathrm{SW}$ when waves attain an average height of 2-3 $\mathrm{m}$ with a pe- 

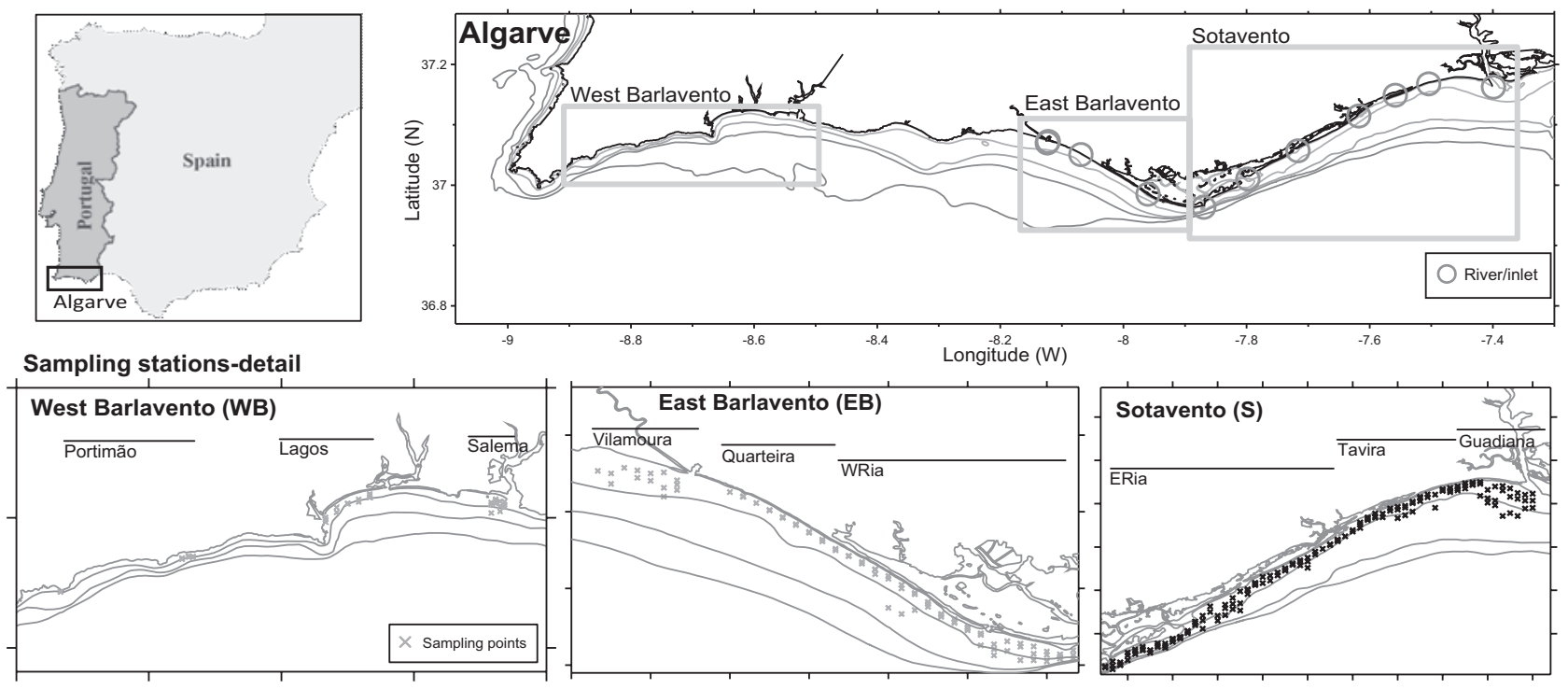

FIG. 1. - Location of the sampling sites and respective areas/sub-areas (on the top of the map). Sub-sector transects in Sotavento: Guadiana, around the delta of the Guadiana river (transects 1-10); Tavira, from Cacela to Tavira (transects 11-26); and ERia, between Tavira and Olhão (transects 27-58). Sub-sector transects in East Barlavento: WRia, west side of Ria Formosa (transects 59-76); Quarteira, from the the beginning of Ria Formosa coastal lagoon to Quarteira (transect 77-85) and Vilamoura and from Vilamoura to Olhos de Água (transect 86-92). Subsector transects in West Barlavento: Portimão (Delta of the river Arade; transects 93-96); Lagos, between Alvor and Lagos (transects 97-101) and Salema, between Salema and Zavial (transects 102-108). Beach exposure is indicated by the width of the line: thicker line corresponds to higher exposure (E-SE direction) and thinner line to lower exposure (NE direction).

riod of 7-8 s (Pires, 1989; Moura et al., 2006, and ref. therein). The sampled area is characterised by sediments with a large range of grain sizes, from muddy areas on the outflow of the Guadiana river to the coarse sand found on western grounds. The most recent description of the sediments of the south coast of Portugal was made in 1986 (Moita, 1986).

The objectives of the present paper are: 1) to provide comprehensive data on species occurrence and abundance of the megabenthic bivalve soft-bottom coastal assemblages in S Portugal; and 2) to relate the geographical and bathymetric patterns described with ecological factors such as beach exposure, river proximity and burying depth.

\section{MATERIAL AND METHODS}

\section{Study area and sampling}

Bivalve surveys were carried out between 2000 and 2007 in both spring and autumn, except in 2003 (only spring was sampled) and 2004 (only autumn was sampled), with the RV Diplodus. During these surveys the sampling design was kept unchanged. The coast was subdivided into 108 transects perpendicular to the coastline, with a distance of $1 / 2$ nautical mile between each other (Fig. 1). Each transect was established between 3 and $15 \mathrm{~m}$ depth, comprising 8 sampling stations distanced $1.8 \mathrm{~m}$ from each other (A: $3 \mathrm{~m}$; B: $4.8 \mathrm{~m}$; C: 6.6;m; D: 8.4 m; E: 10.2 m; F: 12 m; G: 13.8 m; H: 15.6 m). In each transect 2 to 4 stations were randomly chosen. During fishing operations, two dredges were used simultaneously, one adapted to razor clam fishing (dredge tooth length of $35 \mathrm{~cm}$ ) and one designed for clam fishing (dredge tooth length of $20 \mathrm{~cm}$ ); mesh size on both dredges was $25 \mathrm{~mm}$. Further details on the dredges used can be found in Gaspar (1996; 2003). Dredges were towed for 5 min at a mean speed of 1.5 knots, sweeping an area of $144 \mathrm{~m}^{2}$. Once onboard, the catch from each of the dredges was placed in a labelled plastic bag. The samples were later processed in the laboratory, where the bivalve species were identified to species level, counted, measured and weighed. In the present study data from 8600 samples were analysed. Species names followed CLEMAM nomenclature.

For the description of geographical patterns, the coast was divided into three sectors (Fig. 1): Sotavento (S) (from $7.3^{\circ} \mathrm{W}$ to $7.88^{\circ} \mathrm{W}$ longitude; transects 1-58), East Barlavento (EB) (from $7.88^{\circ} \mathrm{W}$ to $8.42^{\circ} \mathrm{W}$ longitude; transects $59-92$ ) and Western Barlavento (WB) (from $8.42^{\circ} \mathrm{W}$ to $9.00^{\circ} \mathrm{W}$ longitude; transects 93-108) (Gaspar, 1996; Gaspar et al., 1999a; Gaspar et al., 2002b; Gaspar et al., 2003). For a finer geographic approach, these three sectors of the coast were further subdivided. Sotavento sec- 
tor was subdivided into three sub-areas: 1) Guadiana, around the delta of the Guadiana river (transects 1-10); 2) Tavira, from Cacela to Tavira (transects 11-26); and 3) ERia, between Tavira and Olhão (transects 27-58). The EB sector was also subdivided into three sub-areas: 4) WRia, West side of Ria Formosa (transects 59-76); 5) Quarteira, from the beginning of Ria Formosa coastal lagoon to Quarteira (transect 77-85); and 6) Vilamoura, from Vilamoura to Olhos de Água (transect 86-92). In the WB sector, sampling was carried out in three separate areas: off 7) Portimão (Delta of the river Arade, transects 9396); 8) Lagos, between Alvor and Lagos (transects 97-101); and 9) Salema, between Salema and Zavial (transects 102-108).The location of all sectors and sub-sectors is shown in Figure 1.

Beach exposure was calculated for the EB and $\mathrm{S}$ coastal sectors. Thus, the successive azimuths of the $3 \mathrm{~m}$ bathymetric line were estimated and for each point an exposure index was calculated, in agreement with the coast geographical direction (NE, E and SE) (Nunes, 2007). According to the exposure index estimated (NE coast corresponded to a 0.47 exposure, E to a 0.99 and SE to a 0.96 exposure), the area was divided into high (E or SE direction, respectively transects 1-14, 49-53 and 60-92) and low (NE direction, transects 15-48 and 54-59) beach exposure.

The river influence was determined by visually selecting sedimentation areas surrounding the river inlets/mouths, using both Google Earth (http://earth. google.com/) and aerial ortho-photomaps of the coast, for the EB and S Sectors. The sites outside/inside these areas were classified as having a low/high river influence, respectively. Neither river influence nor beach exposure was estimated for the WB area, due to the sparseness of sampling points (this area is not continuous sandy beach like the others, but it is intercepted by rocky cliffs).

\section{Data analysis}

All species caught were classified according to their global occurrence over the 7 years of sampling into rare (species occurring less than 30 times during all surveys, i.e. $\mathrm{N}<30$ or less than $0.35 \%$ of occurrence) and common $(\mathrm{N}>30)$. For the commoner species the percentage occurrence, median density and significant differences across factors analysed, i.e. dredge type, season, beach exposure and river influence (whether species occurred significantly more in any factor level than in the remaining ones, i.e. showed a preference), were estimated. The median was used (instead of the mean) due to the nonGaussian highly skewed nature of the data (typical in such datasets). Percentage occurrence was calculated as the number of times the species occurred in the respective factor level, divided by the number of samples in the factor. Significant differences in occurrences were tested by applying a chi-squared test on the number of times the species occurred in each factor level, and using the total number of samples within each factor level as prior probability.

Cluster analysis using the Bray-Curtis distance matrix and the average agglomeration method was applied to sub-areas and depth matrices of species occurrences, in order to identify the main patterns in species assemblages in relation to depth and geography. Species occurrence within each of the main clusters was calculated in relation to the environmental factor. All analyses were performed using the R-project software (R-project, 2006). Multivariate methods were applied using the vegan package (Oksanen et al., 2007).

\section{RESULTS}

During the 7 years of bivalve surveys on the fishable grounds of the Algarve coast, 75 megabenthic bivalve species were identified, representating 25 families and 7 orders (Tables 1 and 2, Appendix 1). From these, 48\% (36 species) occurred less than 30 times over the 8600 hauls $(\sim 0.3 \%)$ and were thus considered rare species for the area/depth sampled in the study (Table 1). Most of these occurred fewer than 10 times (26 species, $0.1 \%$ occurrence), with 12 appearing only once. Among the 36 less common species, 11 occurred only in the Sotavento area, 8 in Sotavento/ East Barlavento and 4 only in East Barlavento. No species occurred exclusively in West Barlavento. In relation to the dredge, 9 species were captured only with bivalve dredge and 8 species with the razor shell dredge (Table 1$)$.

A more detailed analysis was performed for the most common bivalves ( $52 \%$ of the species), i.e. species occurring more than 30 times. Among these, the commonest species were Chamelea gallina, Spisula solida, Mactra stultorum, Acanthocardia tuberculata and Donax trunculus. These bivalves occurred more than 2000 times (i.e. in $21 \%$ of the samples). Six species occurred 1000-2000 times and fourteen 
TABLE 1. - Bivalve species that occurred fewer than 30 times in the surveys carried out between 2000 and 2006 along the south coast of Portugal. N, total occurrences; Density, median density; Max, maximum abundance; Dredge, number of times that a species was collected in the $\mathrm{CD}$ (clam dredge) or RD (razor shell dredge); Season, number of times that a species was collected in a specific season (S, spring and A: autumn); Area, area of occurrence (WB, West Barlavento; EB, East Barlavento; and S, Sotavento; see the location of the sectors in Fig. 1); Depth, occurrence depth (A: 3 m; B: 4.8 m; C: 6.6;m; D: 8.4 m; E: 10.2 m; F: 12 m; G: 13.8 m; H: 15.6 m).

\begin{tabular}{|c|c|c|c|c|c|c|c|c|}
\hline Code & Species & $\mathrm{N}$ & Density & Max & Dredge & Season & Area & Depth \\
\hline Abr alb & Abra alba & 8 & 0.012 & 0.028 & $4 \mathrm{~B} 4 \mathrm{E}$ & $7 \mathrm{~S} 1 \mathrm{~A}$ & $2 \mathrm{~S} 6 \mathrm{~EB}$ & A-E G \\
\hline Aca des & Acanthocardia deshayesii & 14 & 0.007 & 0.035 & 10B 4E & $14 \mathrm{~A}$ & $8 \mathrm{~S} 6 \mathrm{WB}$ & A-E \\
\hline Aeq com & Aequipecten commutatus & 1 & 0.007 & 0.007 & 1B & $1 \mathrm{~A}$ & $\mathrm{~S}$ & $\mathrm{~F}$ \\
\hline Aeq ope & Aequipecten opercularis & 5 & 0.012 & 0.023 & $4 \mathrm{~B} 1 \mathrm{E}$ & $4 \mathrm{~S} 1 \mathrm{~A}$ & $\mathrm{~S}$ & $\mathrm{DE}$ \\
\hline Bar can & Barnea candida & 1 & 0.007 & 0.007 & $1 \mathrm{E}$ & $1 \mathrm{~A}$ & EB & $\mathrm{B}$ \\
\hline Cer gla & Cerastoderma glaucum & 1 & 0.007 & 0.007 & $1 \mathrm{~B}$ & $1 \mathrm{~S}$ & $\mathrm{~S}$ & B \\
\hline Chl var & Chlamys varia & 1 & 0.012 & 0.012 & $1 \mathrm{E}$ & $1 \mathrm{~A}$ & $\mathrm{~S}$ & E \\
\hline Dip rot & Diplodonta rotundata & 9 & 0.012 & 0.058 & 3B 6E & $4 \mathrm{~S} 5 \mathrm{~A}$ & $8 \mathrm{~S} 1 \mathrm{~EB}$ & D E-G \\
\hline Don ven & Donax venustus & 1 & 0.021 & 0.021 & $1 \mathrm{~B}$ & $1 \mathrm{~S}$ & $\mathrm{~EB}$ & B \\
\hline Ens arc & Ensis arcuatus & 16 & 0.007 & 0.035 & $4 \mathrm{~B} 12 \mathrm{E}$ & $1 \mathrm{~S} 15 \mathrm{~A}$ & 8S 6WB2EB & B-E G \\
\hline Gar dep & Gari depressa & 2 & 0.009 & 0.012 & $2 \mathrm{~B}$ & $1 \mathrm{~S} 1 \mathrm{~A}$ & $1 \mathrm{~S} 1 \mathrm{WB}$ & DH \\
\hline Gou min & Gouldia minima & 3 & 0.007 & 0.028 & 2B 1E & $1 \mathrm{~S} 2 \mathrm{~A}$ & $\mathrm{~S}$ & $\mathrm{CE}$ \\
\hline Lim exi & Lima exillis & 2 & 0.007 & 0.007 & $2 \mathrm{~B}$ & $1 \mathrm{~S} 1 \mathrm{~A}$ & $\mathrm{~S}$ & $\mathrm{CF}$ \\
\hline Luc div & Lucinella divaricata & 1 & 0.007 & 0.007 & 1B & $1 \mathrm{~A}$ & $\mathrm{~EB}$ & $\mathrm{D}$ \\
\hline Lyo nor & Lyonsia norwegica & 13 & 0.012 & 0.028 & $9 \mathrm{~B} 4 \mathrm{E}$ & $5 \mathrm{~S} 8 \mathrm{~A}$ & $12 \mathrm{~S} 1 \mathrm{WB}$ & $\mathrm{C}-\mathrm{G}$ \\
\hline Mac bal & Macoma balthica & 1 & 0.012 & 0.012 & $1 \mathrm{E}$ & $1 \mathrm{~A}$ & $\mathrm{~S}$ & $\mathrm{C}$ \\
\hline Mac mel & Macoma melo & 30 & 0.012 & 0.081 & 14 & 16 & 6EB24S & A-H \\
\hline Mod bar & Modiolus barbatus & 3 & 0.007 & 0.012 & $3 \mathrm{E}$ & $3 \mathrm{~A}$ & $2 \mathrm{~S} 1 \mathrm{~EB}$ & BEH \\
\hline Mod sp & Modiolus sp. & 20 & 0.012 & 0.023 & 13B 7E & $3 \mathrm{~S} 17 \mathrm{~A}$ & $18 \mathrm{~S} 2 \mathrm{~EB}$ & A C-H \\
\hline Mod sub & Modiolarca subpicta & 1 & 0.012 & 0.012 & 1B & $1 \mathrm{~S}$ & $\mathrm{~S}$ & E \\
\hline Mys und & Mysia undata & 6 & 0.012 & 0.012 & $3 \mathrm{~B} 3 \mathrm{E}$ & $2 \mathrm{~S} 4 \mathrm{~A}$ & $5 \mathrm{~S} 1 \mathrm{WB}$ & CDEG \\
\hline Myt gal & Mytilus galloprovincialis & 21 & 0.023 & 0.208 & $13 \mathrm{~B} 8 \mathrm{E}$ & 14S 7A & 13S 1WB7EB & A-H \\
\hline Nuc min & Nuculana minuta & 7 & 0.012 & 0.046 & 5B 2E & $5 \mathrm{~S} 2 \mathrm{~A}$ & $6 \mathrm{~S} 1 \mathrm{WB}$ & BCEF \\
\hline Pec max & Pecten maximus & 23 & 0.012 & 0.069 & 14B 9E & $2 \mathrm{~S} 21 \mathrm{~A}$ & 12S 1WB10EB & A C-H \\
\hline Pin fra & Pinna fragilis & 16 & 0.012 & 0.18 & $8 \mathrm{~B} 8 \mathrm{E}$ & $16 \mathrm{~A}$ & $15 \mathrm{~S} 1 \mathrm{~EB}$ & A D-H \\
\hline Sol sco & Solecurtus scopula & 3 & 0.007 & 0.014 & $3 \mathrm{E}$ & $2 \mathrm{~S} 1 \mathrm{~A}$ & 1S $2 \mathrm{~EB}$ & C-E \\
\hline Sol str & Solecurtus strigilatus & 1 & 0.007 & 0.007 & $1 \mathrm{E}$ & $1 \mathrm{~S}$ & EB & $\mathrm{C}$ \\
\hline Tap rho & Tapes rhomboides & 23 & 0.007 & 0.069 & 17B $6 \mathrm{E}$ & $11 \mathrm{~S} 12 \mathrm{~A}$ & 18S 3WB2EB & B-H \\
\hline Tel dis & Tellina distorta & 1 & 0.012 & 0.012 & $1 \mathrm{E}$ & $1 \mathrm{~A}$ & $\mathrm{~S}$ & $\mathrm{H}$ \\
\hline Tel don & Tellina donacina & 1 & 0.012 & 0.012 & 1B & $1 \mathrm{~A}$ & $\mathrm{~S}$ & $\mathrm{H}$ \\
\hline Tel fab & Tellina fabula & 4 & 0.007 & 0.007 & 1B 3E & $1 \mathrm{~S} 3 \mathrm{~A}$ & 1S 3EB & $\mathrm{A}-\mathrm{C} \mathrm{E}$ \\
\hline Tel nit & Tellina nitida & 6 & 0.007 & 0.014 & 3B 3E & $2 \mathrm{~S} 4 \mathrm{~A}$ & $5 \mathrm{~S} 1 \mathrm{~EB}$ & C E-G \\
\hline Ven aur & Venerupis aurea & 1 & 0.035 & 0.035 & $1 \mathrm{E}$ & $1 \mathrm{~A}$ & $\mathrm{~S}$ & $\mathrm{H}$ \\
\hline Ven cas & Venus casina & 8 & 0.007 & 0.007 & $8 \mathrm{~B}$ & $5 \mathrm{~S} 3 \mathrm{~A}$ & 3S 2WB3EB & D E-G \\
\hline Ven sen & Venerupis senegalensis & 6 & 0.017 & 0.056 & 3B 3E & $4 \mathrm{~S} 2 \mathrm{~A}$ & 3S 1WB2EB & B-E \\
\hline Ven ver & Venus verrucosa & 13 & 0.012 & 0.046 & $8 \mathrm{~B} 5 \mathrm{E}$ & $10 \mathrm{~S} 3 \mathrm{~A}$ & 10S 1WB2EB & B E-H \\
\hline
\end{tabular}

species 100-1000 times. The remaining fourteen species, less than 100 times. The species that showed greatest density were Donax trunculus followed by Chamelea gallina, Spisula solida, Acanthocardia paucicostata and Spisula subtruncata (Table 2).

\section{Burying depth}

Two different dredges were used: one specific for razor clams, which has larger teeth and thus buries deeper into the substrate, and one for clams, equipped with shorter teeth and catching mainly species closer to the sediment surface. Thus, bivalve occurrence in each dredge may be used an indirect indicator of the maximum burying-depth of the species, by comparing the species occurrence for both dredges.

From the 39 species showing greatest occurrences, 13 species (33\%) occurred similarly in both dredges, 15 species $(38 \%)$ were significantly more frequent in the clam dredge (CD), i.e. closer to the sediment surface, and the other 11 species (28\%) were significantly more frequent in the razor clam dredge (RD), suggesting that these species burrow deeper into the sediment (Fig. 2 and Table 2). All species belonging to the Pharidae and Solenidae families (razor shells) and most Tellinidae were significantly more frequent in the RD dredge, whilst the families Cardiidae and Donacidae were significantly more frequent in the CD dredge.

\section{Season}

Although most bivalve species occurred similarly in both seasons ( $19 \mathrm{sp} ; 49 \%$ ), a large proportion were more abundant in the autumn surveys (17 sp., 44\%), whereas only three species were commoner during the spring surveys (Fig. 3 and Table 2). Most species belonging to the families Cardiidae and Mactridae were commoner in the autumn surveys (6 out of 7 , 
TABLE 2. - Total occurrence (N, i.e. the number of samples where the species was identified), median density (Density), maximum density (Max) of the bivalve species occurring more than 30 times during all surveys on the south coast of Portugal. The columns Dredge, Season and Sector indicate the percentage occurrence, median abundance (in parenthesis) and preference (significance tested with chi-squared $*$ p-value $>0.05$, ** p-value $<0.01)$ of these bivalve species. Dredge (CD, for clam dredge and RD for the razor shells dredge), season (spring and autumn surveys). Sector: S, Sotavento; EB, East Barlavento; and WB, West Barlavento (see Fig. 1 for location).

\begin{tabular}{|c|c|c|c|c|c|c|c|c|c|}
\hline \multirow[t]{2}{*}{ Code } & \multirow[t]{2}{*}{ Species } & \multirow[t]{2}{*}{$\mathrm{N}$} & \multirow[t]{2}{*}{ Density } & \multirow[t]{2}{*}{ Max } & \multicolumn{2}{|c|}{ Dredge } & \multicolumn{2}{|c|}{ Season } & \multirow[t]{2}{*}{ Sector } \\
\hline & & & & & CD & RD & Spr & Aut & \\
\hline Aca acu & Acanthocardia aculeata & 491 & 0.012 & 0.69 & $5.6(0.01)$ & $5.9(0.01)$ & $4(0.01)^{* *}$ & $7.4(0.01)^{* *}$ & S-WB \\
\hline Aca ech & Acanthocardia echinata & 32 & 0.023 & 0.266 & $0.4(0.04)$ & $0.3(0.02)$ & $0.2(0.01)^{* *}$ & $0.5(0.03) * *$ & $\mathrm{~S}$ \\
\hline Aca pau & Acanthocardia paucicostata & 178 & 0.046 & 1.426 & $2.4(0.05)^{* *}$ & $1.7(0.05)^{* *}$ & $1.9(0.05)$ & $2.3(0.06)$ & $\mathrm{S}$ \\
\hline Aca spi & Acanthocardia spinosa & 93 & 0.007 & 0.058 & $1.1(0.01)$ & $1(0.01)$ & $0.7(0.01)^{* *}$ & $1.5(0.01)^{* *}$ & WB-S \\
\hline Aca tub & Acanthocardia tuberculata & 2303 & 0.014 & 0.949 & $29.4(0.02)^{* *}$ & $24.1(0.01)^{* *}$ & $20.4(0.01)^{* *}$ & $33.4(0.01)^{* *}$ & WB \\
\hline Ano eph & Anomia ephippium & 97 & 0.023 & 0.532 & $1.2(0.03)$ & $1.1(0.01)$ & $1.2(0.02)$ & $1(0.01)$ & $\mathrm{S}$ \\
\hline Arc cra & Arcopagia crassa & 76 & 0.007 & 0.127 & $0.7(0.01)^{* *}$ & $1.1(0.01)^{* *}$ & $1(0.01)$ & $0.8(0.01)$ & S-EB \\
\hline Cal chi & Callista chione & 555 & 0.012 & 0.405 & $6(0.01)$ & $6.9(0.01)$ & $6.7(0.01)$ & $6.2(0.01)$ & WB \\
\hline Cer edu & Cerastoderma edule & 33 & 0.007 & 0.062 & $0.5(0.01)$ & $0.3(0.01)$ & $0.1(0.01)^{* *}$ & $0.6(0.01)^{* *}$ & S-EB \\
\hline Cha gal & Chamelea gallina & 3864 & 0.104 & 35.403 & $46.5(0.14)^{* *}$ & $43.4(0.08)^{* *}$ & $44.3(0.09)$ & $45.6(0.12)$ & S-EB \\
\hline Cha str & Chamelea striatula & 225 & 0.014 & 0.428 & $2.9(0.02)$ & $2.3(0.01)$ & $2.7(0.02)$ & $2.5(0.01)$ & WB-S \\
\hline Chl fle & Chlamys flexuosa & 35 & 0.012 & 0.046 & $0.6(0.01)^{* *}$ & $0.3(0.01)^{* *}$ & $0.3(0.01)$ & $0.5(0.01)$ & $\mathrm{S}$ \\
\hline Cla bro & Clausinella brongniartii & 214 & 0.012 & 0.201 & $2.7(0.01)$ & $2.3(0.01)$ & $2.2(0.01)$ & $2.8(0.01)$ & $\mathrm{EB}$ \\
\hline Cla fas & Clausinella fasciata & 99 & 0.007 & 0.127 & $1.4(0.01)^{* *}$ & $0.9(0.01)^{* *}$ & $1(0.01)$ & $1.3(0.01)$ & $\mathrm{EB}$ \\
\hline Cor gib & Corbula gibba & 77 & 0.012 & 0.498 & $1.3(0.01)^{* *}$ & $0.5(0.01)^{* *}$ & $0.7(0.01)$ & $1.1(0.01)$ & $\mathrm{S}$ \\
\hline Don sem & Donax semistriatus & 1580 & 0.021 & 1.014 & $20.9(0.02) * *$ & $15.9(0.02)^{* *}$ & $22.2(0.02)^{* *}$ & $14.4(0.01)^{* *}$ & EB-S \\
\hline Don tru & Donax trunculus & 2057 & 0.118 & 4.385 & $24.8(0.14)$ & $23(0.1)$ & $24.3(0.11)$ & $23.5(0.12)$ & EB-S \\
\hline Don var & Donax variegatus & 783 & 0.014 & 1.023 & $10.1(0.01)^{* *}$ & $8.1(0.01)^{* *}$ & $6.3(0.01)^{* *}$ & $12(0.01)^{* *}$ & S-EB \\
\hline Don vit & Donax vittatus & 209 & 0.007 & 0.188 & $3(0.01)^{* *}$ & $1.8(0.01)^{* *}$ & $2.4(0.01)$ & $2.5(0.01)$ & $\mathrm{EB}$ \\
\hline Dos exo & Dosinia exoleta & 774 & 0.012 & 1.124 & $5.8(0.01)^{* *}$ & $12.2(0.01)^{* *}$ & $9.7(0.01)^{* *}$ & $8.3(0.01)^{* *}$ & EB-S \\
\hline Dos lup & Dosinia lupinus & 507 & 0.012 & 0.347 & $4.7(0.01)^{* *}$ & $7.1(0.01)^{* *}$ & $5.5(0.01)$ & $6.3(0.01)$ & $\mathrm{S}$ \\
\hline Ens ens & Ensis ensis & 134 & 0.007 & 0.032 & $0.8(0.01)^{* *}$ & $2.3(0.01)^{* *}$ & $1.2(0.01)^{* *}$ & $1.9(0.01)^{* *}$ & WB-EB \\
\hline Ens sil & Ensis siliqua & 1897 & 0.022 & 7.361 & $12.3(0.01)^{* *}$ & $31.8(0.04)^{* *}$ & $21.2(0.02)$ & $22.9(0.02)$ & EB-S \\
\hline Gar fer & Garifervensis & 109 & 0.007 & 0.167 & $1.6(0.01)^{* *}$ & $1(0.01)^{* *}$ & $0.8(0.01)^{* *}$ & $1.8(0.01)^{* *}$ & S-WB \\
\hline Lae cra & Laevicardium crassum & 1140 & 0.014 & 4.429 & $14.8(0.01)^{* *}$ & $11.7(0.01)^{* *}$ & $6.6(0.01)^{* *}$ & $20.1(0.02)^{* *}$ & WB-S \\
\hline Lut ang & Lutraria angustior & 331 & 0.013 & 2.849 & $1.4(0.01)^{* *}$ & $6.3(0.01)^{* *}$ & $1.5(0.01)^{* *}$ & $6.3(0.01)^{* *}$ & $\mathrm{~S}$ \\
\hline Mac stu & Mactra stultorum & 1028 & 0.01 & 0.443 & $9.4(0.01)^{* *}$ & $14.5(0.01)^{* *}$ & $10.4(0.01)^{* *}$ & $13.6(0.01)^{* *}$ & EB-WB \\
\hline Mac gla & Mactra glauca & 3231 & 0.023 & 1.469 & $38.5(0.02)$ & $36.6(0.02)$ & $33.7(0.03)^{* *}$ & $41.6(0.02)^{* *}$ & $\mathrm{~S}$ \\
\hline Mod adr & Modiolus adriaticus & 59 & 0.012 & 0.243 & $1(0.01)^{* *}$ & $0.4(0.01)^{* *}$ & $0.3(0.01)^{* *}$ & $1(0.01)^{* *}$ & $\mathrm{~S}$ \\
\hline Ost edu & Ostrea edulis & 70 & 0.014 & 0.278 & $0.9(0.01)$ & $0.7(0.01)$ & $0.8(0.02)$ & $0.8(0.01)$ & S-EB \\
\hline Pan ina & Pandora inaequivalvis & 182 & 0.012 & 1.1 & $2.7(0.01)^{* *}$ & $1.6(0.01)^{* *}$ & $2.4(0.01)$ & $1.8(0.01)$ & $\mathrm{S}$ \\
\hline Pha leg & Pharus legumen & 1860 & 0.028 & 59.423 & $13.2(0.02)^{* *}$ & $30.1(0.04)^{* *}$ & $19.2(0.03)^{* *}$ & $24.1(0.03)^{* *}$ & EB-S \\
\hline Pit rud & Pitar rudis & 59 & 0.023 & 0.386 & $0.8(0.01)$ & $0.6(0.02)$ & $0.8(0.02)$ & $0.6(0.01)$ & $\mathrm{S}$ \\
\hline Sol mar & Solen marginatus & 68 & 0.007 & 0.183 & $0.2(0.01)^{* *}$ & $1.4(0.01)^{* *}$ & $0.8(0.01)$ & $0.7(0.01)$ & S-EB \\
\hline Spi sol & Spisula solida & 3402 & 0.083 & 70.905 & $41.8(0.1)^{* *}$ & $37.3(0.06)^{* *}$ & $36.7(0.06)^{* *}$ & $42.5(0.1)^{* *}$ & S-EB \\
\hline Spi sub & Spisula subtruncata & 1837 & 0.035 & 40.79 & $23.1(0.04)^{* *}$ & $19.6(0.04)^{* *}$ & $18.5(0.04)^{* *}$ & $24.3(0.04)^{* *}$ & EB-S \\
\hline Tel inc & Tellina incarnata & 95 & 0.007 & 0.097 & $0.6(0.01)^{* *}$ & $1.6(0.01)^{* *}$ & $1.2(0.01)$ & $1(0.01)$ & S-EB \\
\hline Tel ten & Tellina tenuis & 601 & 0.014 & 1.739 & $5.4(0.01)^{* *}$ & $8.6(0.01)^{* *}$ & $8.3(0.01)^{* *}$ & $5.6(0.01)^{* *}$ & $\mathrm{~EB}-\mathrm{S}$ \\
\hline Thr pap & Thracia papyracea & 70 & 0.007 & 0.936 & $0.7(0.01)$ & $0.9(0.01)$ & $0.4(0.01)^{* *}$ & $1.2(0.01)^{* *}$ & EB-WB \\
\hline
\end{tabular}

and 5 out of 5 , respectively). All remaining bivalve families showed no seasonal trend.

\section{Spatial distribution, beach exposure and river influence}

All species showed significant differences in the occurrences between the areas, except Cerastoderma edule, Chamelea striatula, Garifervensis and Thracia papyracea (Fig. 3A). All species occurred in the Sotavento sector, whereas Pitar rudis and Acanthocardia paucicostata were not found in EB, Anomia ephippium and Chlamys flexuosa were not present in WB. Species belonging to the family Cardiidae were preferably found in WB and $\mathrm{S}$, whereas the families Donacidae, Mactridae and Tellinidae occurred mainly in the EB and S coastal sectors.
Cluster analysis identified four main groups of species with particular affinities according to geographic distribution (Fig. 3B). Group 1 contains 13 species that occurred mainly in the Guadiana outflow and vicinities; Group 2 contains 4 species that occurred mainly in Vilamoura and Tavira; Group 3 contains three species that occurred mainly in Salema and Lagos; and Group 4 contains 22 species distributed along the entire coast. The results of the cluster analysis are in agreement with Figure $3 \mathrm{~A}$.

Eleven species (28\%) showed no significant statistical differences in function of beach exposure (Fig. 3 and Table 2), whereas eight (21\%) were commoner in low exposed beaches and $20(51 \%)$ species occurred significantly more in exposed areas. Species belonging to Mactridae and Veneridae were more frequent on more exposed beaches and tellinids preferred non- 


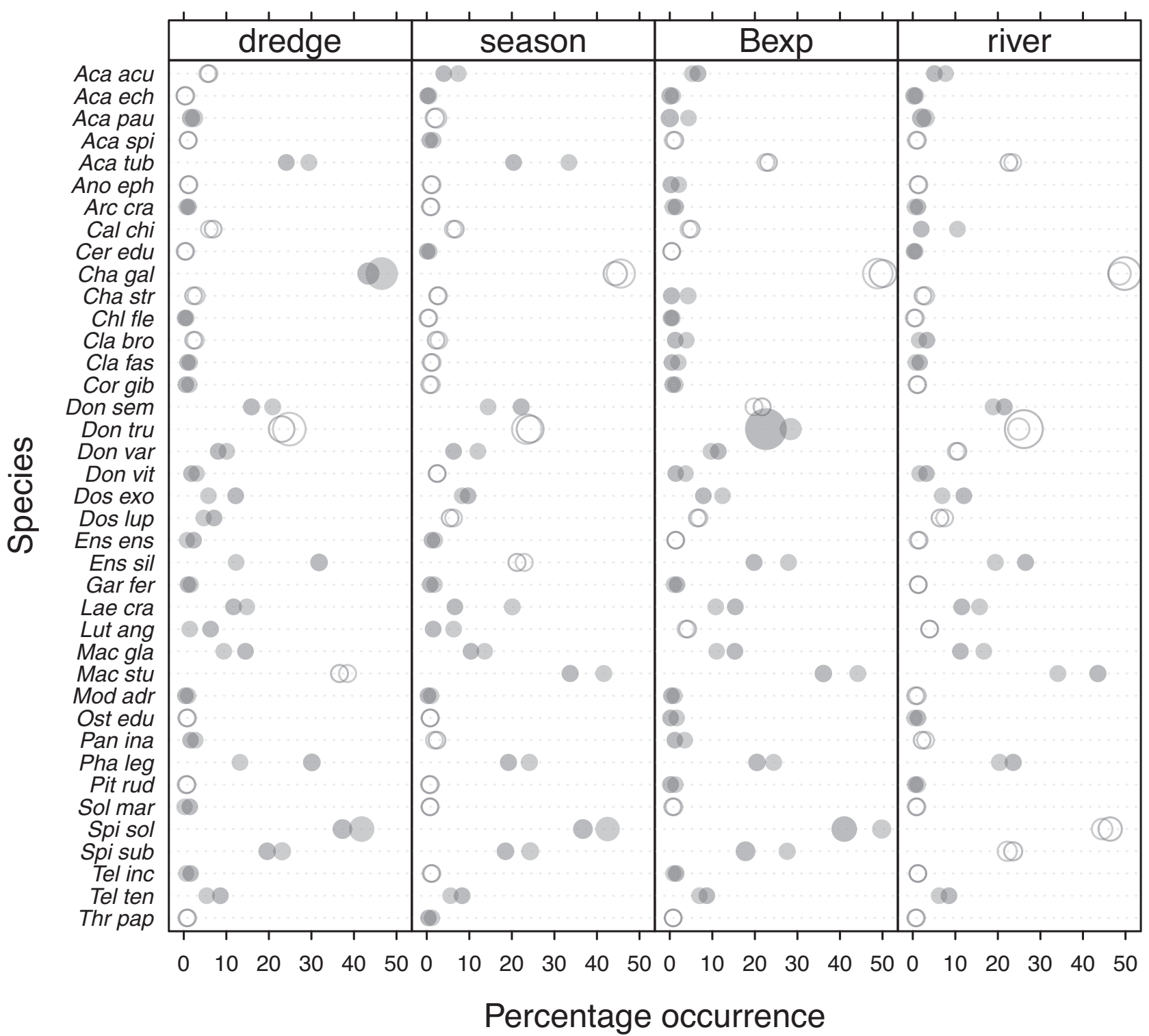

FIG. 2. - Species occurrence and respective median abundance (diameter of the circles) according to dredge type (darker grey for razor clam dredge and lighter grey for clam dredge), season (dark grey for autumn and light grey for spring surveys), beach exposure ('Bexp', dark grey for higher exposure and light grey for less exposed) and river influences ('river', dark grey for higher influence and light grey for less influence). Both beach exposure (high and low) and river influences (high and low) were calculated only for the East Barlavento and Sotavento areas. The location of beach exposure levels and the main rivers is shown in Fig. 1. Open circles indicate non-significant deviations, tested with chi-squared test. See Table 2 for complete species names.

exposed coasts. Regarding river influence, 20 species $(51 \%)$ were not significantly influenced by the distance to the river mouths, 11 species $(28 \%)$ were more frequent outside the vicinity of river mouths and eight $(21 \%)$ were significantly more common within the river sedimentation area (Fig. 2).

\section{Bathymetry}

Overall, shallower species with a modal depth at 3-6 m (Donax trunculus, Spisula solida, Ensis siliqua, Mactra stultorum and Chamelea gallina) showed greater occurrences and abundances than the deeper ones (Fig. 4). Bathymetric distribution was clearly unimodal in most species, although Spisula solida, Tellina tenuis, Dosinia exoleta, Thracia papyracea, Donax variegatus and Clausinella fasciata appeared to have a second peak in bathymetric distribution.

In general the depth distribution patterns observed did not change between seasons (Table 2, Fig. 4A). Nevertheless, some species (e.g. Anomia ephippium, Acanthocardia paucicostata and A. aculeata) appeared to have a bathymetric shift over the modal class, from depth $\mathrm{E}(10.2 \mathrm{~m})$ in spring 
A

\begin{tabular}{|c|c|c|c|c|}
\hline \multirow{3}{*}{$\begin{array}{l}4 \\
2 \\
0\end{array}$} & Calchi & Laecra & Aca spi & {$[0,0.02]$} \\
\hline & प⿴囗十冖 & 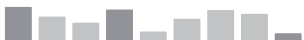 & & $\square \quad(0.05,0.5]$ \\
\hline & Aca tub & Lut ang & Ens ens & Thr pap \\
\hline 0 & $0 \mathrm{lal}$ & 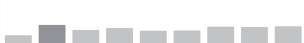 & & \\
\hline & Phaleg & Garfer & Tel inc & Cer edu \\
\hline & $-1=0$ & & & \\
\hline & Don vit & Dos exo & Cla bro & Cla fas \\
\hline & $\mathrm{E}$ & 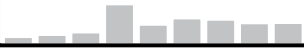 & L & \\
\hline & Spisol & Ens sil & Don sem & Tel ten \\
\hline & 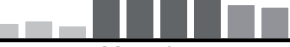 & 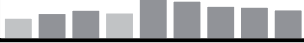 & 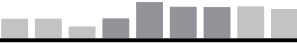 & 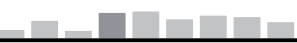 \\
\hline & Mac gla & Don var & Mac stu & Don tru \\
\hline & 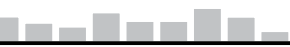 & $\mathrm{L}=\mathrm{n}=\mathrm{n}$ & $\mathrm{an}=\mathrm{a}$ & H \\
\hline & Aca ech & Solmar & Chlffe & Arc cra \\
\hline 2 & & & & E \\
\hline & Pit rud & Ostedu & Cor gib & Mod adr \\
\hline & & & & \\
\hline & Aca $a c u$ & Chastr & Pan ina & Ano eph \\
\hline & $\mathrm{C}=\mathrm{C}$ & 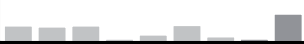 & & 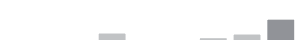 \\
\hline & Chagal & Spi sub & Dos lup & Aca pau \\
\hline & 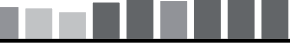 & 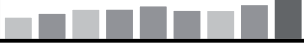 & $\mathrm{C}$ & 1 \\
\hline
\end{tabular}

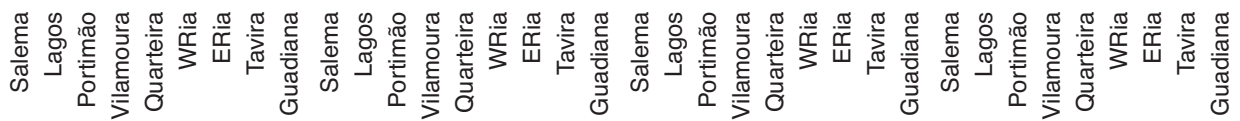

Geographic sub-sector

B

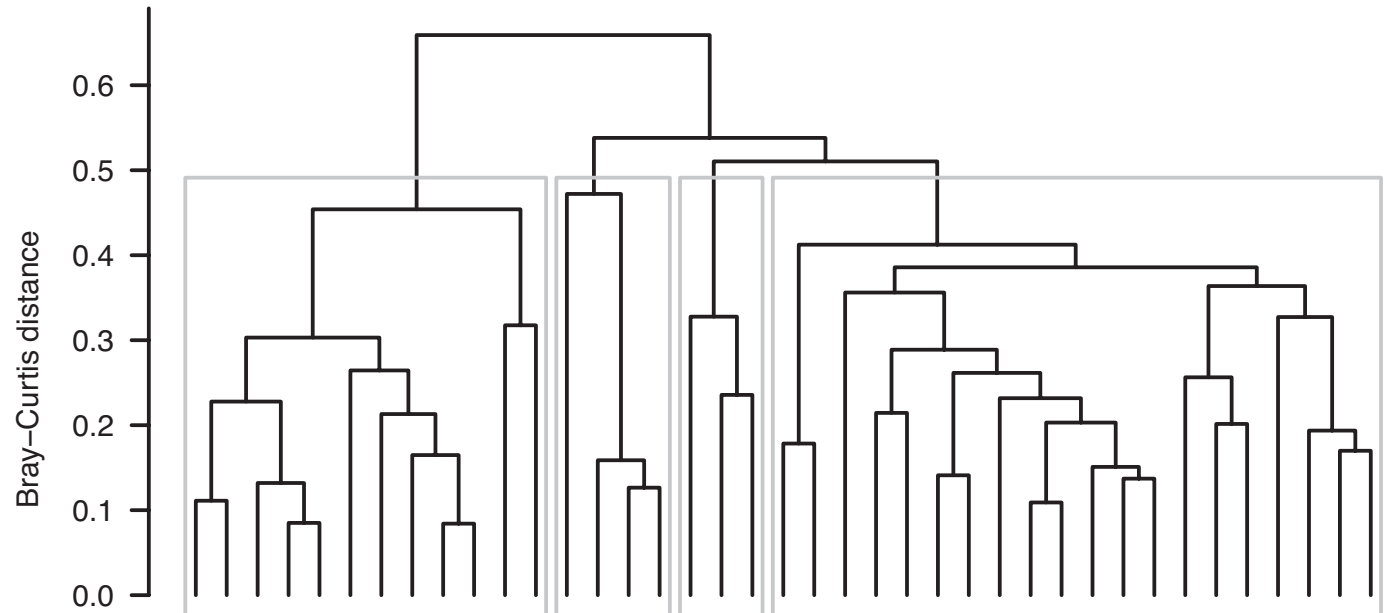

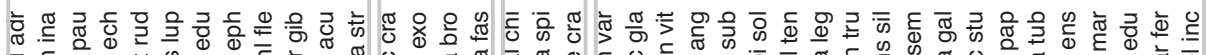

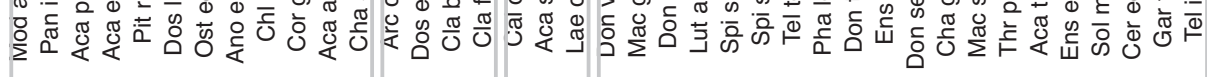

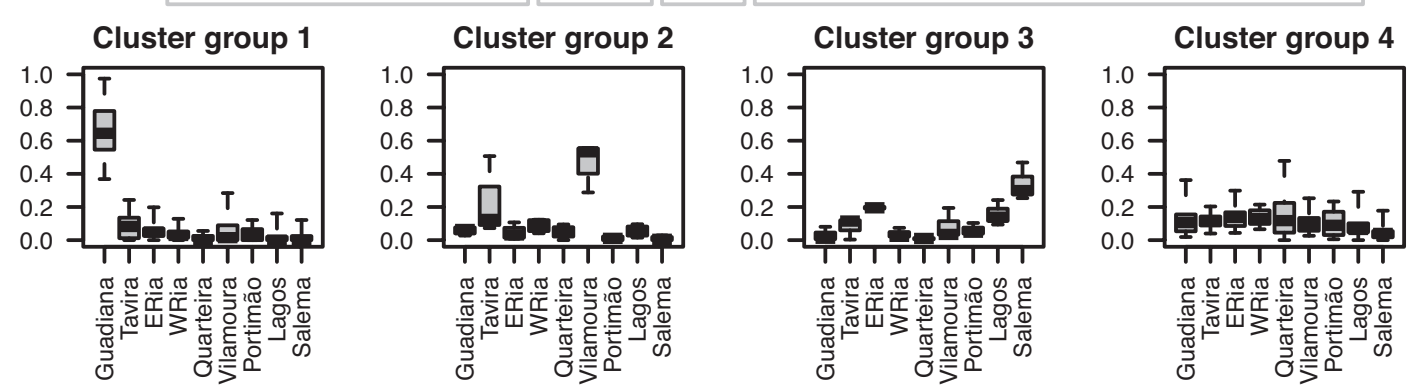

FIG. 3. - A, bivalve species occurrence (\%) across the sub-areas on the south coast of Portugal (see the text for further details). The colour of the bars corresponds to species median density $\left(\mathrm{N} . \mathrm{m}^{-2}\right)$, which can be seen in the top right corner. B, cluster analysis of species/subareas standardised matrices and respective boxplots for the cluster composition. See Table 2 for complete species names. 


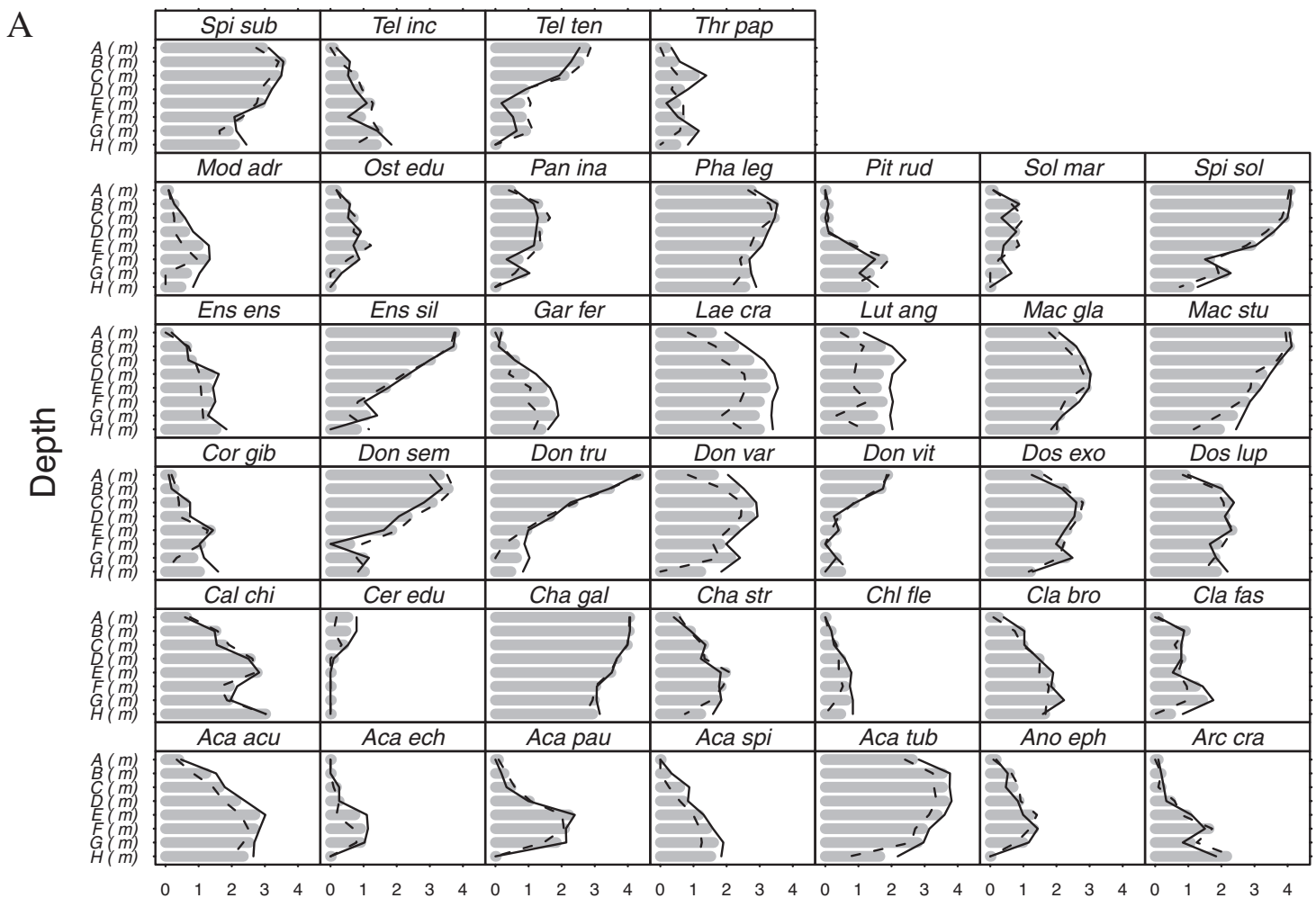

B

$\log \%$ ocurrence

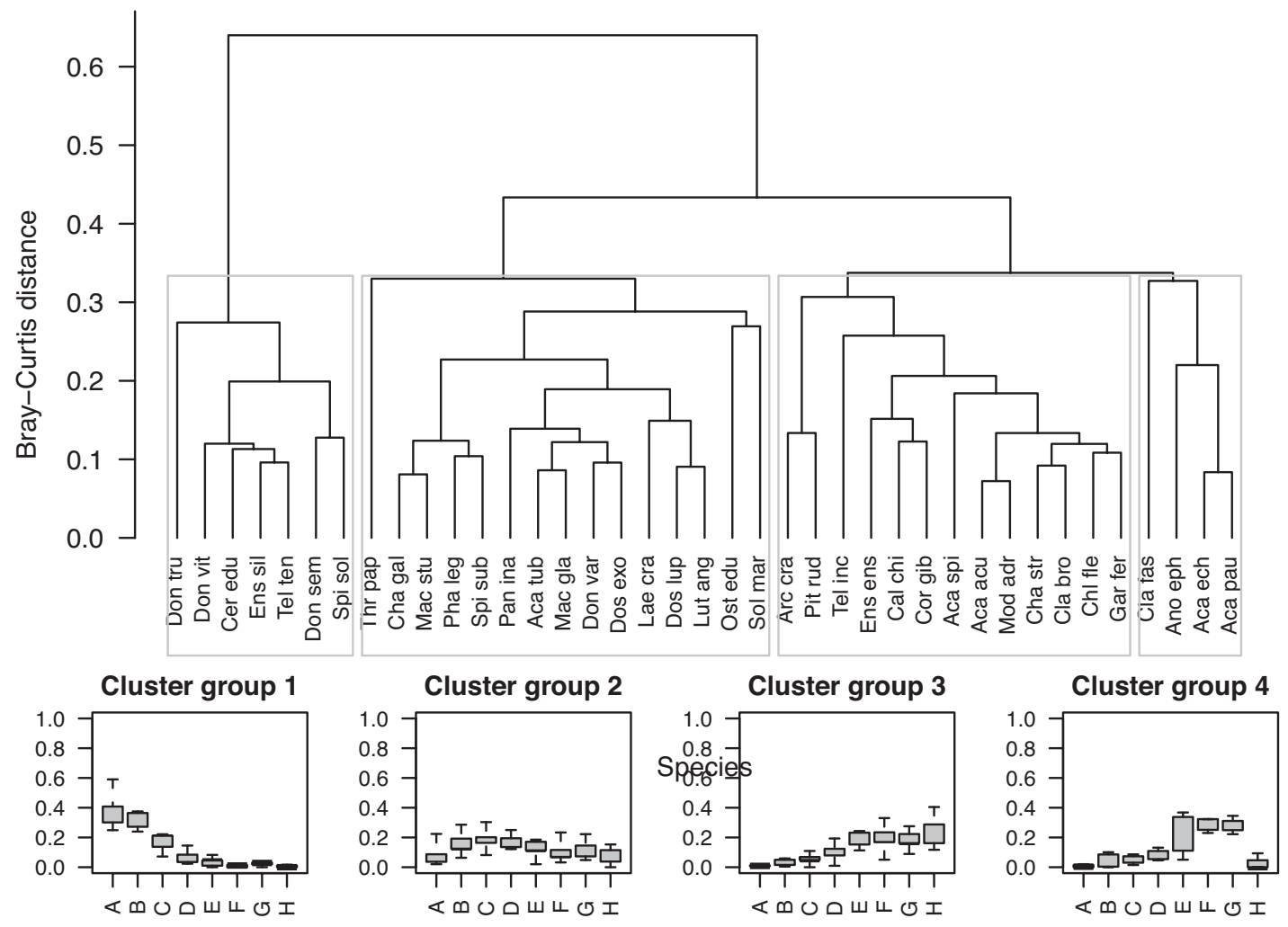

FIG. 4. - A, bivalve species occurrence $(\log \%+1)$ by depth interval. The bars represent overall occurrence and the lines show seasonal occurrences (continuous line for autumn surveys; dashed line for spring surveys). The plots are ordered first by species modal depth, then by maximum level of occurrence. B, cluster analysis of species/depths intervals standardised matrices and respective boxplots for the clusters composition. See Table 2 for complete species names. 

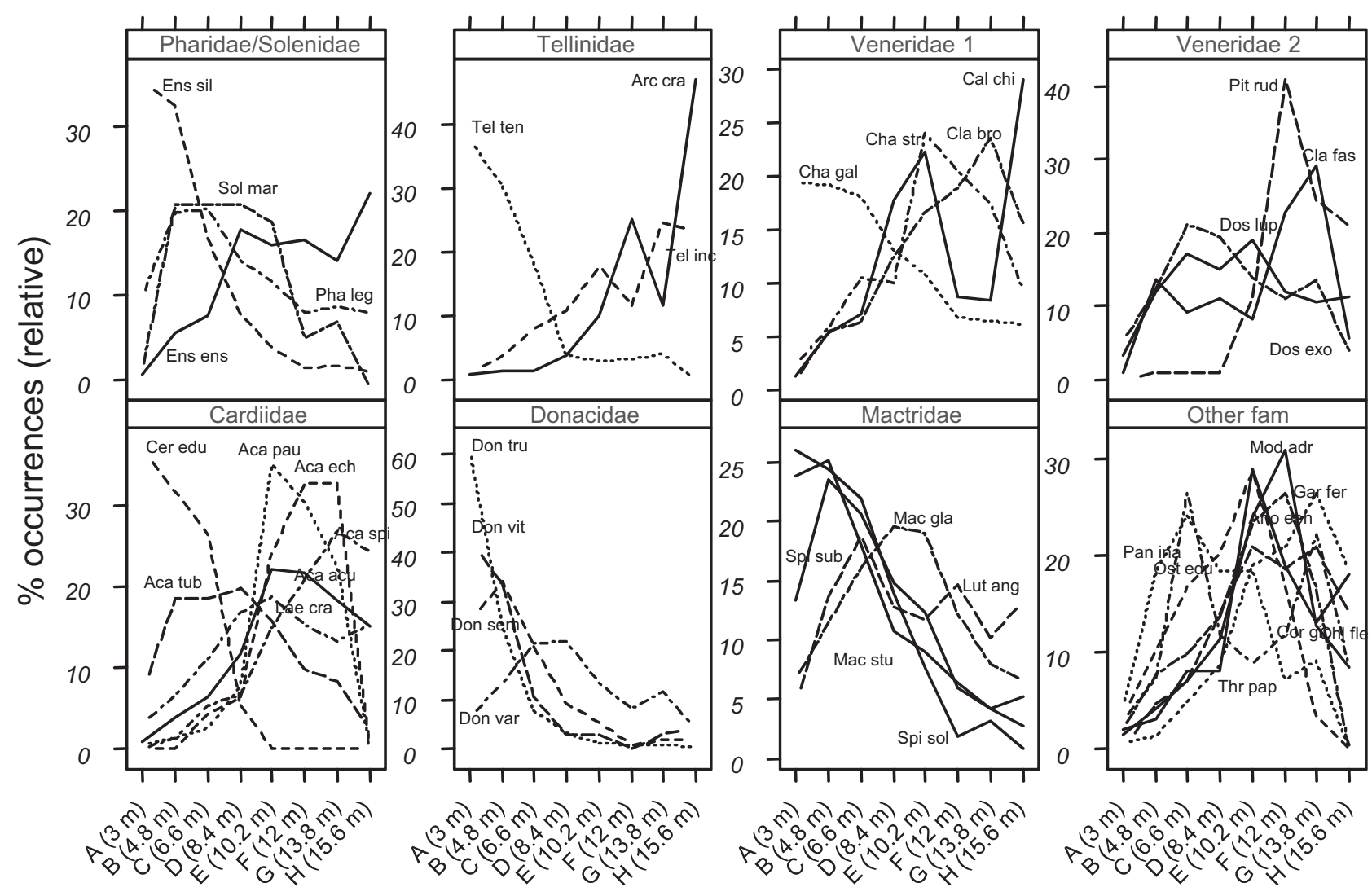

\section{Depth intervals}

FIG. 5. - Bathymetric distribution of the most common species by family. See Table 2 for complete species names.

to depth F (12 m) in autumn. Some species showed a similar occurrence pattern in spring and autumn surveys, whereas others appeared to undertake some kind of seasonal bathymetric migration. Both occurrence peaks of Solen marginatus were deeper in the autumn surveys (from 4.8/8.4 $\mathrm{m}$ in spring to 6.6/10.2 $\mathrm{m}$ in autumn). Spisula elliptica showed greater occurrences at 4.8/6.6 m in autumn and at 3/10.2 m in spring. Chamelea striatula peaks were shallower in autumn and appeared to occur deeper in spring, similarly to Anomia ephippium, Pandora inaequivalvis and Ostrea edulis (Table 2, Fig. 4A).

Four main groups were identified by cluster analysis applied to the bathymetric distributions (Fig. 4B). Cluster 1 contained 6 species that occurred mainly in shallow waters, between 3 and 6.6 m (A-C) water depth, Cluster 2 contained 17 species that showed a wide depth distribution from 3 to $10.2 \mathrm{~m}$ (A-E), Cluster 3 contained 17 bivalve species that were mostly distributed between 6.6 and 15.6 $m$ depth (D-H) and Cluster 4 contained only two species that mainly occurred between 12 and 15.6 m (F-H) depth.
Donacidae and Mactridae (except Mactra glauca) were represented mostly by shallow species, whereas Cardiidae (except Acanthocardia tuberculata and Cerastoderma edule) were represented mostly by species occurring in deeper waters (Table 2, Fig. 5). Some genera showed a sequential bathymetric distribution of the modal class. For example, the genus Acanthocardia, with relatively large and hard shells, showed a bathymetric sequence of species maximum occurrences, from the shallower A. tuberculata (A-D) followed by A. aculeata (D-F), A. paucicostata (E-G, with maximum at E) and A. echinata (E-G, with maximum at $\mathrm{G}$ ) towards the deepest species, A. spinosa $(\mathrm{G}-\mathrm{H})$. The species belonging to the genus Donax, were all shallow species, but also showed sequential maximum occurrences, from shallower to deeper distribution: D. trunculus, $D$. vittatus, D. semistriatus and D. variegatus. Within the razor clams species, Ensis siliqua was the shallower species (mainly A-B), followed by Pharus legumen (mainly B-C) and Ensis ensis in deeper waters $(\mathrm{C}-\mathrm{H})$, although with large differences in 
occurrences. In the family Tellinidae, the shallow Tellina tenuis was replaced by $T$. incarnata and Arcopagia crassa in deeper waters. The families Mactridae and Veneridae, the most important in number of species, formed a more heterogeneous group. Chamelea gallina and C. striatula are two closely resembling species; the former, inhabiting shallower waters (maximum occurrences at $4.8 \mathrm{~m}$ ), is distributed mainly in the Mediterranean, whereas the latter occurrs mainly in deeper waters (maximum occurrences at $10.2 \mathrm{~m}$ ) and is commoner in the northeastern Atlantic. Among the venerids, Callista chione has the largest and hardest shell, and this species occurs in deeper waters, peaking both at 10.2 and at $15.6 \mathrm{~m}$ depth. Both Dosinia exoletalD. lupinus and Clausinella fasciata/C. brongniartii showed very similar depth distributions, although the first genus was shallower than the second one. Within Mactridae, all Spisula species and Mactra stultorum showed a similar shallower depth distribution, followed by Mactra glauca, peaking at 8.4-10.2 m. However, the occurrences of these species were largely different, i.e. Spisula solida showed a maximum of $56 \%$ occurrences, S. subtruncata $23 \%$ and $S$. elliptica only $3 \%$. Similarly, Mactra stultorum maximum occurrences were $56.8 \%$.

In the remaining species belonging to other families, Corbula gibba, Ostrea edulis and Anomia ephippium showed similar bathymetric distributions, occurring mainly from 10.2 to $13.8 \mathrm{~m}$ depth. The large and thin species Gari fervensis (Family Psammobiidae) and Lutraria angustior (Family Mactridae), which have similar shapes, showed a distinct bathymetric distribution, with the former occurring in shallow waters and the latter occurring in deeper waters. The fragile Pandora inaequivalvis showed a shallow distribution.

\section{DISCUSSION}

The coastal area under study constitute a passage from the Atlantic to the Mediterranean, thus representing a bio-geographic transition in which changes in species composition and abundance are of particular importance. The existence of a well -documented upwelling close to the western part of the sampling area is expected to be reflected in the abundance of most coastal species (Erzini, 2005). Most species identified (Acanthocardia aculeata,
Acanthocardia paucicostata, Acanthocardia tuberculata, Callista chione, Clausinella fasciata, , Chamelea striatula, Donax variegatus, Dosinia lupinus, Ensis siliqua, Ensis ensis, Laevicardium crassum, Lutraria lutraria, Modiolus adriaticus, Modiolus sp., Ostrea edulis, Pandora inaequivalvis, Spisula elliptica, Spisula solida, Tellina fabula, Tellina incarnata and Tapes rhomboides) have their northern geographic limit of distribution in Norway, Iceland or the British Isles and have their southern limit near Morocco or in the Iberian Peninsula. The remaining species (Anomia ephippium, Arcopagia crassa, Cerastoderma edule, Corbula gibba, Donax trunculus, Donax vittatus, Dosinia exoleta, Gari fervensis, Lutraria angustior, Pharus legumen, Solen marginatus, Spisula subtruncata and Tellina tenuis) have a wider geographic distribution, and are present from the northern hemisphere (Norway, Iceland and the UK) to central Africa. Other species (Acanthocardia spinosa, Donax semistriatus, Donax venustus, Flexopecten flexuosa, Gouldia minima, Macoma melo, Mytilus galloprovincialis and Venus casina) are found in the Iberian Peninsula, including the Mediterranean Sea. Chamelea gallina is found mainly in the Mediterranean, having its limit in the Algarve (Backeljau et al., 1994; Rufino et al., 2007). Finally, Pitar rudis is a more tropical species, being distributed from Spain to Angola and in the Mediterranean Sea (Macedo et al., 1999).

Between 2000 and 2007, 75 megabenthic bivalve species were caught in the dredging along the Algarve coast. Of these, $48 \%$ occurred fewer than 30 times, which may be related to aspects such as the sampling techniques (e.g. small specimens are certainly under-estimated), bathymetry (e.g. some species in the current work have their bathymetric limits deeper from the $15 \mathrm{~m}$ surveyed) and/or depth in the sediment (deeper buriers were probably underestimated), type of substrate (e.g. Mytilus galloprovincialis, as well as Ostrea edulis and Anomia ephippium typically occur in rocky areas, which were not covered in the current surveys) and/or specimen fragility (e.g. the strong metal dredges can severely damage thinner/more fragile shells such as those of Barnea candida and Tellina spp.). Chícharo et al. (2002), sampling with quadrats sieved through a $1 \mathrm{~mm}$ mesh (for macrofauna) and corers (for meiofauna) at 7-9 m depth off Lagos and Vilamoura (western coast of the Algarve), identified 38 bivalve species, among which 7 were not collected in the current study. Within the commonest species, occurring in $>21 \%$ of the samples, were the commer- 
cial species Chamelea gallina, Spisula solida, Donax trunculus and Ensis siliqua. This result was expected since the surveys were designed to evaluate the stock status of these species and within the surveyed area these species are the most common bivalves.

Burying depth into the sediment has important consequences for species ecology (Zwarts, 1986). Living closer to the sediment surface may increase the susceptibility of animals to predators and to being flushed away. However, it is also advantageous: suspension feeding is more efficient for physical reasons and deposit feeding is more profitable as a longer part of the siphon can be stretched over the surface, thus increasing the feeding radius (De Goeij and Luttikhuizen, 1998, and ref. therein). For these reasons, the depth at which a bivalve is buried appears to be a compromise between the risks of starvation and predation, but it is also affected by oxygen deficiency and sediment characteristics (Zwarts, 1986; Zwarts and Wanink, 1989; Tallqvist, 2001). Also, as burying depth is actively controlled by the animal, it may change between areas, seasons and environmental gradients (De Goeij and Luttikhuizen, 1998). Overall, about one third of the species were captured with both dredges; one third was caught with $\mathrm{CD}$ so they must be less buried; and the remaining species were caught mainly with the RD dredge so they must be buried more deeply in the sediment.

In the current study, species from the families Cardiidae and Donacidae were mostly shallow burrowers. Species belonging to the former family have a rounded/globose shell shape, with fairly small siphons and a strong muscular foot; they avoid predators by jumping and some species may even show a limited swimming capability (Savazzi, 1985). Further, Cardiidae shell sculpture may increase/facilitate anchoring in the sediment (Watters, 1994), thus avoiding the problem of being flushed away easily. Donacidae species are small and flat-shaped and have relatively elongated shells, being able to live within the swash zone (Huz et al., 2002). Donacids were found mainly in a shallower bathymetry $(<10$ $m$ depth). They occupied the swash area, thus being subject to tides and desiccation, although their rapid burying allows them to avoid the problems associated with this type of habitat (McLachlan and Young, 1982). Most species from the family Tellinidae and all species from the families Pharidae and Solenidae were more frequently caught by the RD dredge. In the case of tellinids, the species observed in the catches have characteristically thin and fragile shells, so burying deeper could provide an extra protection. Species from the other two families, Pharidae and Solenidae, have elongated shells which easily dig into the sediment like a razor blade. Urban (1994) concluded that species with an elongated shape, such as Ensis spp., spend less energy in burrowing, being adapted to deeper burying.

Most bivalve species did not show marked seasonal differences. Seventeen species were commoner in the autumn surveys and three in the spring surveys (44\% mainly in autumn, $49 \%$ in both seasons and $8 \%$ mainly in spring). Brazeiro and Defeo (1996) observed that beach species tended to occupy the upper levels of their distributional areas in spring-summer and the lower levels in autumn-winter. Therefore, it is possible that the greater abundance of some species in autumn is related to species occupying the lower limit of their distribution. Seasonal changes in burying depth, which have been reported for some bivalve species (Zwarts and Wanink, 1989), may also cause such differences. For example, the increase in storm events in autumnwinter can lead to both changes in burying behaviour and sediment movements that cause differences in species catchability. Neuberger Cywiak et al. (1990) concluded that the migration observed in Donax semistriatus to the beach upper zone took place as a result of heavy storms and currents. Some species can perform seasonal bathymetric migrations while others are known to perform migrations during their life cycle, from a couple of metres to many kilometres (Hiddink et al., 2002; Hiddink and Wolff, 2002; Gibson, 2003). Recruitment could also influence seasonal differences in bivalve abundance. Still, the life cycles are studied mainly for commercial species in the current area: Chamelea striatula spawning occurs between April and early August and Donax trunculus between March and August (Gaspar and Monteiro, 1998; Gaspar et $a l ., 1999 b)$. Both species occurred significantly more in the spring surveys of the present study, which correspond to the spawning season.

Most of the species studied were commoner in more exposed coastal areas than in less exposed areas. Most previous studies focus on beaches whose limits are not exactly the ones of the study area, but as they are a part of it, some features could be comparable (McLachlan and Dorvlo, 2007a, b). Bivalves from reflective beaches were found to be uniformly small, medium- to fast-burrowing, wedge-shaped and of high density, whereas those from more dissipative beaches vary widely in all these characteristics (McLachlan et al., 1995). The three beaches charac- 
terised in terms of morphology on the south coast of Portugal all showed a reflective profile (Garão, Faro and Culatra), with wave heights of 0.34-0.8 m and sediment grain sizes of $0.26-0.38 \mathrm{~mm}$ (Ciavola et al., 1997). These three beaches probably represent an intermediate profile within the study area, but no further study was found. Towards the Guadiana river, as the grain size decreases the beaches may be more dissipative. In one way, more exposed beaches might have a greater turnover of nutrients, which is favourable to suspensivorous species, but higher beach exposure results in a more hydrodynamic environment and therefore lower sediment stability of the sediment (i.e. species can be flushed away).

Twenty-eight percent of the species were significantly more frequent outside areas of river influence. The influence of river presence on the south coast of Portugal is more important in the case of the Guadiana River, which is the most important one in the study area and showed the highest bivalve diversity (Rufino et al., 2008). River flow rate is known to influence the bivalve communities nearby. Ambrogi and Ambrogi (1987) observed the highest recruitment of Spisula subtruncata in years with the greatest river flow rates, whereas Schoeman and Richardson (2002) observed that recruits of Donax serra were more abundant in areas further from the main river mouth vicinity. Due to river sedimentation any animal buried in the sediment close to a river mouth will have progressively more sediment above it, especially during rainy periods. Another factor that may interfere is the presence of a water treatment plant in the area (Rufino et al., 2008). Most effluents are discharged into rivers, which transport not only organic matter but also undesirable pollutants that could be harmful to the species living nearby (Cabaço et al., 2008).

All species except Pitar rudis and Acanthocardia paucicostata, which were not found in $\mathrm{EB}$, and Anomia ephippium, Chlamys flexuosa and Macoma melo, which were not present in $\mathrm{WB}$, were found all over the coastal area studied. Cluster analysis classified species according to their occurrence in different geographic sectors, distinguishing 10 species typical from Sotavento, three from WB and six from EB. Nevertheless, the geographic trends observed are related to environmental differences between areas rather than to biogeographic trends. Of the 10 species occurring mainly in $\mathrm{S}$, most were found around the outflow area of the Guadiana river (these species were not commoner within the river vicinity, but were specifically found in the Guadiana). The Guadiana delta has the largest extension of muddy sediments on this part of the Portuguese coast and most of these species (Acanthocardia aculeata, A. paucicostata, Anomia ephippium, Corbula gibba, Dosinia lupinus, Pandora inaequivalvis and Pitar rudis) are probably typical inhabitants of this type of sediment. Similarly, the area between Vilamoura and Quarteira has coarser sediments, which corresponded to larger occurrences of three species typical of this sediment type (Clausinella brongniartii, Clausinella fasciata and Dosinia exoleta). Arcopagia crassa, Donax variegatus and Mactra glauca, which were found preferentially on S coastal sector and were not caught in the muddier sediments, were also found in large percentages between Vilamoura and Quarteira, where there is also a river outflow (Ribeira de Quarteira). Thus, rivers outflows are probably the primary factor contributing to the presence of these four species.

Depth is an important factor structuring the community (Abelló et al., 2002; McBreen et al., 2008). Although the majority of the species showed a clearly uni-modal bathymetric distribution, some species appeared to have a bimodal distribution in depth (Spisula solida, Tellina tenuis, Dosinia exoleta, Thracia papyracea, Donax variegatus and Clausinella fasciata). The shallowest species also showed the greatest occurrences and abundances, so overall occurrence/abundance decreased with depth. The bathymetric patterns observed did not change greatly between seasons. Furthermore, some species may show a depth segregation between adults and juveniles, as is the case of Donax trunculus (Gaspar et al., 2002a). Therefore, in the case of species with a broad bathymetric distribution in which this phenomenon is observed, the survey design adopted only allows the sampling of a fraction of the population. For example, Lutraria angustior was caught in samples taken below $10.2 \mathrm{~m}$ depth with a mean size of $45 \mathrm{~mm} \mathrm{SL}$, although it is reported in the literature that this species reaches $118 \mathrm{~mm} \mathrm{SL}$ and may occur to a maximum depth of $55 \mathrm{~m}$ (Macedo et al., 1999).

Thus, the area under study shows a large diversity of geographical aspects representing specific environmental characteristic that may influence bivalve species. Among those that have been proven to be important are the river influence (mainly in the Guadiana delta; (Erzini, 2005; Cravo et al., 2006)), the inlets of the Ria Formosa coastal lagoon (Vila-Concejo et al., 2002; Vila-Concejo et al., 
2003; Newton and Mudge, 2005; Vila-Concejo et $a l ., 2006)$, no river/inlet influence, and more/less exposed areas (according to the dominant coastal currents). During the seven years of data presented, some of the factors considered (river/inlet proximity and wave exposure) may have changed their exact values slightly. However, the current work provides an initial approach towards the potential influence of such factors on bivalve abundance and distribution. For a more specific analysis on those relationships, further studies specifically designed for such purpose are required.

Coastal bivalves are economically important for local populations in terms of fisheries resources and tourism, among others. However, these groups are severely impacted by anthropogenic sources, so their conservation and management should be a priority for marine resource managers. The information provided in the current paper about species ecology adds further basic knowledge that can be used to improve the difficult task of preserving the coastal area of the Algarve.

\section{ACKNOWLEDGEMENTS}

The authors would like to thank all participants in the bivalve surveys and in subsequent species identification and measuring, and in particular Tibério Simões, José Luis Sofia and Brás. This work formed part of the MMR post-doc program financed by Fundação para a Ciência e a Tecnologia BPD/14935/2004.

\section{REFERENCES}

Abelló, P., A. Carbonell and P. Torres. - 2002. Biogeography of epibenthic crustaceans on the shelf and upper slope off the Iberian Peninsula Mediterranean coasts: implications for the establishment of natural management areas. Sci. Mar., 66(suppl.2): 183-198.

Ambrogi, R. and A.O. Ambrogi. - 1987. Temporal variations of the secondary production in the marine bivalve Spisula subtruncata off the Po river delta (Italy). Estuar. Coast. Shelf Sci., 1987: 369-379.

Ansell, A.D., C.A. Comely and L. Robb. - 1999. Distribution, movements and diet of macrocrustaceans on a Scottish sandy beach with particular reference to predation on juvenile fishes. Mar. Ecol. Prog. Ser., 176: 115-130.

Backeljau, T., P. Bouchet, S. Gofas and L. de Bruyn. - 1994. Genetic variation, systematics and distribution of the venerid clam Chamelea gallina. J. Mar. Biol. Ass. U.K., 74: 211-223.

Brazeiro, A. and O. Defeo. - 1996. Macroinfauna zonation in microtidal sandy beaches: is it possible to identify patterns in such variable environments? Estuar. Coast. Shelf Sci., 42: 523-536.

Cabaço, S., R. Machás, V. Vieira and R. Santos. - 2008. Impacts of urban wastewater discharge on seagrass meadows (Zostera noltii). Estuar. Coast. Shelf Sci., 78: 1-13.

Chícharo, L., J. Regala, M. Gaspar, F. Alves and A. Chícharo. -
2002. Macrofauna spatial differences within clam dredge-tracks and their implications for short-term fishing effect studies. Fish. Res., 54: 349-354.

Ciavola, P., R. Taborda, O. Ferreira and J.A. Dias. - 1997. Field observations of sand-mixing depths on steep beaches. Mar. Geol., 141: 147-156.

Costa, M. - 1994. Agitação marítima na costa portuguesa. An. Inst. Hid. Lisb., 13: 35-40.

Cravo, A., M. Madureira, H. Felicia, F. Rita and M.J. Bebianno. - 2006. Impact of outflow from the Guadiana River on the distribution of suspended particulate matter and nutrients in the adjacent coastal zone. Estuar. Coast. Shelf Sci., 70: 63-75.

De Goeij, P. and P. Luttikhuizen. - 1998. Deep-burying reduces growth in intertidal bivalves: field and mesocosm experiments with Macoma balthica. J. Exp. Mar. Biol. Ecol., 228: 327-337.

Defeo, O. and A. McLachlan. - 2005. Patterns, processes and regulatory mechanisms in sandy beach macrofauna: a multi-scale analysis. Mar. Ecol. Prog. Ser., 295: 1-20.

Dexter, D.M. - 1992. Sandy beach community structure: the role of exposure and latitude. J. Biogeogr., 19: 59-66.

Erzini, K. - 2005. Trends in NE Atlantic landings (southern Portugal): identifying the relative importance of fisheries and environmental variables. Fish. Oceanogr., 14: 195-209.

Fugi, T. - 2007. Spatial patterns of benthic macrofauna in relation to environmental variables in an intertidal habitat in the Humber estuary, UK: Developing a tool for estuarine shoreline management. Estuar. Coast. Shelf Sci., 75: 101-119.

Gaspar, M.B. - 1996. Bivalves do litoral oceânico algarvio. Aspectos da biologia, ecologia e das pescarias dos mananciais de interesse económico: aplicacão à gestão dos recursos. Ph.D. thesis, Univ. Algarve.

Gaspar, M.B. and C.C. Monteiro. - 1998. Reproductive cycles of the razor clam Ensis siliqua and the clam Venus striatula off Vilamoura, southern Portugal. J. Mar. Biol. Assoc. U.K., 78: 1247-1258.

Gaspar, M.B., M. Castro and C.C. Monteiro. - 1999a. Effect of tooth spacing and mesh size on the catch of the Portuguese clam and razor clam dredge. ICES J. Mar. Sci., 56: 103-110.

Gaspar, M.B., R. Ferreira and C.C. Monteiro. - 1999b. Growth and reproductive cycle of Donax trunculus L., (Mollusca: Bivalvia) off Faro, southern Portugal. Fish. Res., 41: 309-316.

Gaspar, M.B., L.M. Chícharo, P. Vasconcelos, A. Garcia, A.R. Santos and C.C. Monteiro. - 2002a. Depth segregation phenomenon in Donax trunculus (Bivalvia: Donacidae) populations of the Algarve coast (southern Portugal). Sci. Mar., 66: 111-121.

Gaspar, M.B., F. Leitao, M.N. Santos, M. Sobral, L. Chícharo, A. Chícharo and C.C. Monteiro. - 2002b. Influence of mesh size and tooth spacing on the proportion of damaged organisms in the catches of the Portuguese clam dredge fishery. ICES J. Mar. Sci., 59: 1228-1236.

Gaspar, M.B., F. Leitao, M.N. Santos, L. Chícharo, M.D. Dias, A. Chícharo and C.C. Monteiro. - 2003. A comparison of direct macrofaunal mortality using three types of clam dredges. ICES J. Mar. Sci., 60: 733-742.

Gibson, R.N. - 2003. Go with the flow: tidal migrations in marine animals. Hydrobiologia, 503: 153-161.

Grémare, A., J.M. Amouroux and G. Vetion. - 1998. Long-term comparison of macrobenthos within the soft bottoms of the Bay of Banyuls-sur-mer (northwestern Mediterranean Sea). J. Sea Res., 40: 281-302.

Guillou, J. and P.G. Sauriau. - 1985. Some observations on the biology and the ecology of a Venus striatula population in the Bay of Douarnenez, Brittany. J. Mar. Biol. Assoc. U.K., 65: 889-900.

Harriague, A., L. Gaozza, A. Montella and C. Misic. - 2006. Benthic communities on a sandy Ligurian beach (NW Mediterranean). Hydrobiologia, 571: 383-394.

Hiddink, J.G. and W.J. Wolff. - 2002. Changes in distribution and decrease in numbers during migration of the bivalve Macoma balthica. Mar. Ecol. Prog. Ser., 233: 117-130.

Hiddink, J., R. Kock and W. Wolff. - 2002. Active pelagic migrations of the bivalve Macoma balthica are dangerous. Mar. Biol. (N Y), 140: 1149-1156.

Huz, R., M. Lastra and J. Lopez. - 2002. The influence of sediment grain size on burrowing, growth and metabolism of Donax trunculus L. (Bivalvia: Donacidae). J. Sea Res., 47: 85-95.

Laudien, J., M. Rojo, M. Oliva, W. Arntz and S. Thatje. - 2007. Sublittoral soft bottom communities and diversity of Mejillones 
Bay in northern Chile (Humboldt Current upwelling system). Helgol. Mar. Res., 61: 103-116.

Macedo, M.C.C., M.I.C. Macedo and J.P. Borges. - 1999. Conchas Marinhas de Portugal. Ed. Verbo, Lisboa.

McBreen, F., J. Wilson, A. Mackie and C. Nic Aonghusa. - 2008. Seabed mapping in the southern Irish Sea: predicting benthic biological communities based on sediment characteristics. Hydrobiologia, 606: 93-103.

McLachlan, A. - 1990. Dissipative beaches and macrofauna communities on exposed intertidal sands. J. Coast. Res., 6: 57-71.

McLachlan, A. and A. Dorvlo. - 2007a. Global patterns in sandy beach macrobenthic communities. J. Coast. Res., 21: 674-687.

McLachlan, A. and A. Dorvlo. - 2007b. Global patterns in sandy beach macrobenthic communities: Biological factors. J. Coast. Res., 23: 1081-1087.

McLachlan, A. and N. Young. - 1982. Effects of low temperature on the burrowing rates of four sandy beach molluscs. J. Exp. Mar. Biol. Ecol., 65: 275-284.

McLachlan, A., E. Jaramillo, O. Defeo, J. Dugan, A. de Ruyck and P. Coetzee. - 1995. Adaptations of bivalves to different beach types. J. Exp. Mar. Biol. Ecol., 187: 147-160.

Moita, I. - 1986. Carta dos sedimentos superfiais. Notícia explicativa da folha SED 7 e 8 . Instituto Hidrográfico, Lisboa. 18.

Moura, D., L. Albardeiro, C. Veiga-Pires, T. Boski and E. Tigano. - 2006. Morphological features and processes in the central Algarve rocky coast (South Portugal). Geomorphology, 81: 345-360.

Neuberger Cywiak, L., Y. Achituv and L. Mizrahi. - 1990. The ecology of Donax trunculus Linnaeus and Donax semistriatus Poli from the Mediterranean coast of Israel. J. Exp. Mar. Biol. Ecol., 134: 203-220.

Newton, A. and S.M. Mudge. - 2005. Lagoon-sea exchanges, nutrient dynamics and water quality management of the Ria Formosa (Portugal). Estuar. Coast. Shelf Sci., 62: 405-414.

Noy-Meir, I. - 1979. Structure and function of desert ecosystems. Israel J Bot 28:1-19. J. Botany, 28: 1-19.

Nunes, M. - 2007. Hazard assessment in Galé-Olhos de Água sea clifs: a tool for coastal management. Master thesis, University of Portsmouth, Faculty of Science, Department of Geography and Univ. Algarve.

Oksanen, J., R. Kindt, P. Legendre and R.B. O'Hara. - 2007. Vegan: Community Ecology Package version 1.8-5. http://cran.rproject.org/.

Pearson, T. and H.P. Mannvik. - 1998. Long-term changes in the diversity and faunal structure of benthic communities in the northern North Sea: natural variability or induced instability? Hydrobiologia. 375-376: 317-329.

Pires, H.N.O. - 1989. O clima de Portugal, Alguns Aspectos do Clima de Agitação Marítima de Interesse para a Navegação na Costa de Portugal, 34 pp. Instituto Nacional de Meteorologia e Geofísica (INMG). Lisboa

R-project. - 2006. R Development Core Team R: A language and environment for statistical computing. R Foundation for Statistical Computing, Vienna, Austria. ISBN 3-900051-07-0, URL http://www.R-project.org.

Reise, K. - 2002. Sediment mediated species interactions in coastal waters. J. Sea Res., 48: 127-141.

Reyes, J.L., J.T. Martins, J. Benavente, Ó. Ferreira, F.J. Gracia, J.M Alveirinho-Dias and F. López-Aguayo. - 1999. Gulf of Cadiz beaches: A comparative response to storm events Bol. Inst. Esp. Oceanogr., 15: 221-228.

Rufino, M.M., M.B. Gaspar, A.M. Pereira and P. Vasconcelos. 2007. The use of shape to distinguish Chamelea gallina and Chamelea striatula (Bivalvia: Veneridae): linear and geometric morphometric methods. J. Morphol., 267: 1433-1440.

Rufino, M.M., M. Gaspar, F. Maynou and C.C. Monteiro. - 2008. Regional and temporal changes in bivalve diversity in the south coast of Portugal. Est. Coast. Shelf Sci., 80: 517-528.

Savazzi, E. - 1985. Adaptive themes in cardiid bivalves. Neues Jahrbuch für Geologie und Paläontologie Abhandlungen. 170: 291-321.

Schoeman, D.S. and A.J. Richardson. - 2002. Investigating biotic and abiotic factors affecting the recruitment of an intertidal clam on an exposed sandy beach using a generalized additive model. J. Exp. Mar. Biol. Ecol., 276: 67-81.

Simboura, N. and A. Zenetos. - 2002. Benthic indicators to use in Ecological Quality classification of Mediterranean soft bottom marine ecosystems, including a new Biotic Index. Medit. Mar. Sci., 3/2: 77-111.

Tallqvist, M. - 2001. Burrowing behaviour of the Baltic clam Macoma balthica: effects of sediment type, hypoxia and predator presence. Mar. Ecol. Prog. Ser., 212: 183-191.

Teske, P.R. and T. Wooldridge. - 2001. A comparison of the macrobenthic faunas of permanently open and temporarily open/ closed South African estuaries. Hydrobiologia, 464: 227-243.

Thrush, S.F., R.D. Pridmore and J.E. Hewitt. - 1994. Impacts on soft-sediment macrofauna: the effects of spatial variation on temporal trends. Ecol. Appl., 4: 31-41.

Urban, H.J. - 1994. Adaptations of six infaunal bivalve species of Chile: Coexistence resulting from differences in morphology, burrowing depth and substrate preference. Arch. Fish. Mar. Res., 42: 183-193.

Vila-Concejo, A., A. Matias, Ó. Ferreira, C. Duarte and J.M.A. Dias. - 2002. Recent Evolution of the Natural Inlets of a Barrier Island System in Southern Portugal. J. Coast. Res., 36: 741-752.

Vila-Concejo, A., O. Ferreira, A. Matias and J.M.A. Dias. - 2003. The first two years of an inlet: sedimentary dynamics. Cont. Shelf Res., 23: 1425-1445.

Vila-Concejo, A., A. Matias, A. Pacheco, O. Ferreira and J.A. Dias. - 2006. Quantification of inlet-related hazards in barrier island systems. An example from the Ria Formosa (Portugal). Cont. Shelf Res., 26: 1045-1060.

Watters, G.T. - 1994. Form and function of unionoidean shell sculpture and shape (Bivalvia). Am. Malacol. Bull., 11: 1-20.

Zeichen, M.M., S. Agnesi, A. Mariani, A. Maccaroni and G.D. Ardizzone. - 2002. Biology and population dynamics of Donax trunculus L. (Bivalvia: Donacidae) in the South Adriatic Coast (Italy). Est. Coast. Shelf Sci., 54: 971-982.

Zwarts, L. - 1986. Burying depth of the benthic bivalve Scrobicularia plana (da Costa) in relation to siphon-cropping. J. Exp. Mar. Biol. Ecol., 101: 25-39.

Zwarts, L. and J. Wanink. - 1989. Siphon size and burying depth in deposit- and suspension-feeding benthic bivalves. Mar. Biol., 100: $227-240$

Scient. ed.: M. Ramón.

Received July 9, 2008. Accepted June 2, 2009.

Published online December 9, 2009. 
AppendiX I. - Bivalve species caught during the surveys carried out between 2000 and 2006 along the south coast of Portugal.
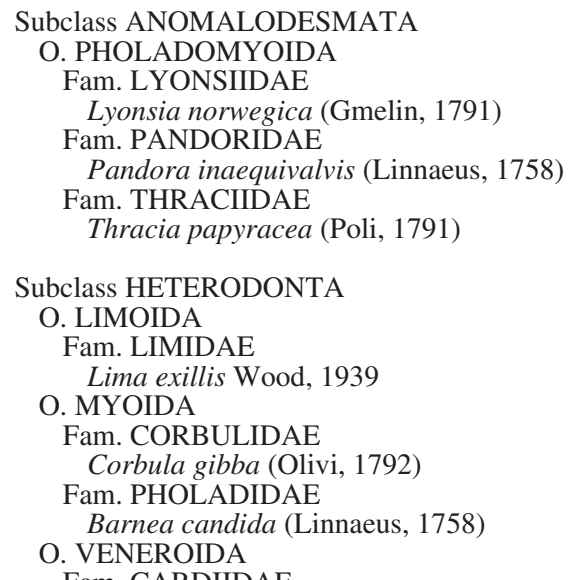

Solecurtus strigilatus (Linnaeus, 1758)

Fam. SOLENIDAE

Solen marginatus (Pulteney, 1799)

Fam. TELLINIDAE

Arcopagia crassa (Pennant, 1777)

Macoma balthica (Linnaeus, 1758)

Macoma melo (Sowerby, 1866)

Tellina distorta (Poli..1791)

Tellina donacina (Linnaeus, 1758)

Tellina fabula (Gmelin, 1791)

Tellina incarnata (Linnaeus, 1758)

Tellina nitida (Poli, 1791)

Tellina tenuis (da Costa, 1778)

Fam. UNGULINIDAE

Diplodonta rotundata (Montagu, 1803)

Fam. VENERIDAE

Callista chione (Linnaeus, 1758)

Chamelea gallina (Linnaeus, 1758)

Chamelea striatula (da Costa, 1778)

Clausinella brongniartii (Payraudeau, 1826)

Clausinella fasciata (da Costa, 1778)

Dosinia exoleta (Linnaeus, 1758)

Dosinia lupinus (Linnaeus, 1758)

Gouldia minima (Montagu, 1803)

Pitar rudis (Poli, 1795)

Venerupis aurea (Gmelin, 1791)

Tapes rhomboides (Pennant, 1777)

Venerupis senegalensis (Gmelin, 1791)

Venus casina (Linnaeus, 1758)

Venus verrucosa (Linnaeus, 1758)

Subclass PALAEOTAXODONTA

O. NUCULOIDA

Fam. NUCULANIDAE

Nuculana minuta (O.F. Müller, 1776)

Subclass PTERIOMORPHIA

O. MYTILOIDA

Fam. MYTILIDAE

Modiolarca subpicta (Cantraine, 1835)

Modiolus adriaticus (Lamarck, 1819)

Modiolus barbatus (Linnaeus, 1758)

Modiolus sp. (Linnaeus, 1758)

Mytilus galloprovincialis (Lamarck, 1819)

O. PTERIOIDIA

Fam. ANOMIIDAE

Anomia ephippium (Linnaeus, 1758)

Fam. PINNIDAE

Pinna fragilis (Pennant, 1777)

Fam. OSTREIDAE

Ostrea edulis (Linnaeus, 1758)

Fam. PECTINIDAE

Aequipecten commutatus (Monterosato, 1875)

Aequipecten opercularis (Linnaeus, 1758)

Chlamys varia (Linnaeus, 1758)

Chlamys flexuosa (Poli, 1795)

Pecten maximus (Linnaeus, 1758) 